\title{
Quantification of Nano-inclusions by EPMA Using Conventional Accelerating Voltages.
}

\author{
$\underline{\text { Claude Merlet }}$
}

GM, CNRS, Université de Montpellier, Place E. Bataillon, 34095 Montpellier, France.

For more than four decades, the electron probe microanalysis (EPMA) has been routinely used to determine the chemical composition of materials on a micrometer scale. For analysis, typical operating conditions use beam energies in a range of 15-20 keV, which give spatial resolutions of about 2-4 $\mu \mathrm{m}$ for a material of mass density of $5 \mathrm{~g} / \mathrm{cm}^{3}$. The volume of interaction can be reducing by decreasing the beam energy, but the large increase in spot size with tungsten gun EPMA at low beam energy limits the lateral resolution to a minimum of about $0.8 \mu \mathrm{m}$ at $7 \mathrm{keV}$. The Schottky emitter field-emission gun (FEG) EPMA now offer the possibility of obtaining a focused electron beam of about $100 \mathrm{~nm}$ with a current of $10 \mathrm{nA}$ at a beam energy of $5 \mathrm{keV}$. At this energy, the penetration range of incident electrons drops to $0.3 \mu \mathrm{m}$, and leads to a significant improvement in the lateral resolution and surface sensitivity, but quantification, at low beam energy is accompanied by experimental and analytical problems which may affect the accuracy of results [1]. Low beam energies require the use of low-energies X-ray lines which are often affected by spectroscopic difficulties and larger uncertainties in the associated massattenuation coefficients (MACs). Moreover, the fluorescence yields are generally lower than for the conventional K-lines, and as a result the detection limit worsens. At low beam energy, as the ratio of beam energy to excitation energy of the lines (overvoltage) is often low, the X-ray intensity decreases significantly. As the X-ray generation is significantly reduced at low beam energy it is necessary to increase the beam current to give a sufficient count rate that can induce damage to the sample. Finally, at low beam energy, a surface layer of several nanometers in thickness represents a much larger fraction of the sample and, therefore, the influence of carbon contamination, surface oxidation, the quality of the sample polish or a conductive coating become more significant.

To reduce these difficulties, another option to quantify small inclusions is to use conventional beam energies in a range of $8-15 \mathrm{keV}$, and to extract by calculation the signal resulting of the inclusion embedded in the surrounding material (matrix). This approach requires firstly that the X-ray intensities coming from the inclusion and the matrix can be calculated and secondly that the surrounding material can be accurately determined. X-ray intensities can be calculated using Monte Carlo simulation [2] or specific analytical program [3]. In this work, to quantify the inclusions, an analytical X-ray distribution in three dimensions was developed. The three dimensions $X$-ray distribution $(F(x, y, z))$ use the product of an X-ray lateral distribution $(\psi(x, y, z))$ and the well known double Gaussian X-ray depth distribution $(\phi(z))$ model, whose accuracy has been largely demonstrated for bulk [4] and thin layers [5]. As the number of ionization is included in the X-ray depth distribution model, the X-ray lateral distribution has to be normalized. In the proposed analytical $F(x, y, z)$ model, the X-ray lateral distribution use a two-dimensional Gaussian distribution with a width depending of the depth. As a consequence the geometry of the X-ray distribution is approaching a truncated sphere (Fig. 1). For a given inclusion geometry and by using this analytical $F(x, y, z)$ model, the X-ray intensities of the inclusion and of the matrix can be computed numerically [6]. 
As, in practice the geometry of the inclusion is unknown, an alternative is to approximate the sample as a cylindrical thin layer with same thickness and radius having the composition of the inclusion in a substrate having the composition of the matrix. The method is an improvement of the multilayer calculation [5], which is done in three dimensions by taking into account the X-ray lateral emission. The algorithm computes by an iterative procedure or by a trial and error approach the emergent intensities corresponding to the various elements of the combination inclusion-matrix and the inclusion size. For the consistency of the results, it is important to have a reasonable convergence between calculation and measurement of the elements of the inclusion but also of the matrix.

To assess the validity of this 3D layer approximation, the method was tested on inclusions embedded in super-alloys, minerals and pure metals for diameters ranging from $100 \mathrm{~nm}$ to $2 \mu \mathrm{m}$. For this study, some samples were built from bulk standards which were crushed to small diameter sizes and embedded in soft metals. Since the compositions of these inclusions are known, these systems were chosen to validate this quantitative method. Some experimental results obtained are presented to demonstrate the validity of this method to perform quantitative analysis of small inclusions. As example, figure 2A shows a BSE map of a geological sample, which contains a large distribution in size of Chromium-spinel inclusion in feldspar [7]. Figure 2B gives the mass concentration of the Chromium-spinel inclusion obtained with the 3D layer approximation in function of the inclusion size; the horizontal lines are the concentrations obtained on sizes larger than 10 microns. The deviation in the concentration is at the maximum $10 \%$ for the magnesium and less that $6 \%$ for the other elements.

\section{References}

[1] C. Merlet and X. Llovet, IOP Conf. Ser. Mater. Sci. Eng. 32 (2012) p. 012016.

[2] R. Gauvin, P. Hovington and D. Drouin, Scanning 17 (1995) p. 202.

[3] O. Arnould and F. Hild, X-Ray Spectrometry 32 (2003) p. 345.

[4] C. Merlet, Mikrochimica Acta, 114/115 (1994) p. 363.

[5] X. Llovet and C. Merlet, Micros. Microanal. 16 (2010) p. 21.

[6] J. T. Armstrong and P. R. Buseck, Anal. Chem. 47 (1975) p. 2148.

[7] F. Kalfoun, D. Ionov and C. Merlet. Earth Planet. Sci. Lett. 199 (2002) p. 49.

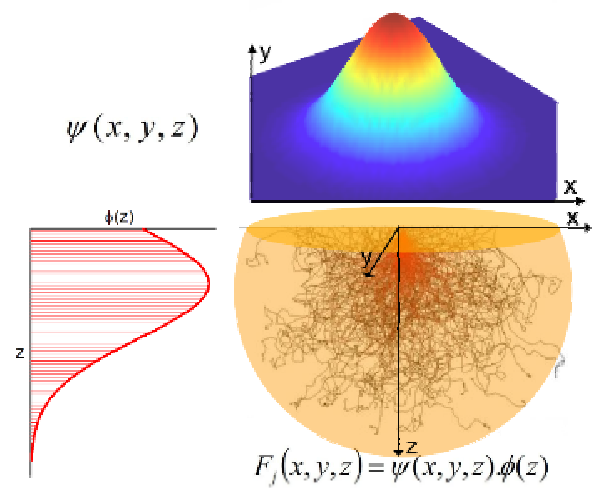

Figure 1. Analytical three-dimensional X-ray distribution $F(x, y, z)$.

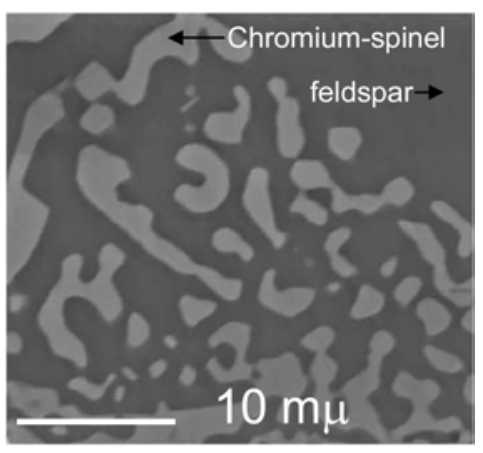

A

Figure 2. (A) BSE map of Chromium-spinel inclusion in feldspar. (B) Weight fraction of inclusions obtained by using the 3D layer approximation. 